\title{
Wandlungen des Gebets im Neuen Testament
}

\author{
Volker Gäckle
}

Es gehört zu den zentralen Fragestellungen der neutestamentlichen Forschung, wie sich die Glaubensinhalte, -formen und -institutionen Israels und des Frühjudentums durch die Offenbarung Gottes im Kommen, Leben, Sterben und Auferstehen Jesu Christi verändert haben. Im Blick auf die kultischen Institutionen des Tempels, des Priestertums und der Opfer wurde dabei forschungsgeschichtlich der Begriff der "Spiritualisierung« bzw. der "Vergeistigung" prägend. Mit ihm wurde ein Prozess beschrieben, im Zuge dessen kultische Terminologie auf neue Sinnbereiche übertragen wurde. ${ }^{1}$ Die Ursprünge dieses Sprachphänomens liegen bereits im Alten Testament (vgl. Ps 50,14; 51,18f; 119,108; 141,2; Jon 2,10) und im Frühjudentum (vgl. z.B. Arist 170.234; Sir 35,1-5; Tob 4,7-12; bBer 26b; 32b; bYev 105a; bMen 110a), als man zunächst nach dem babylonischen Exil und dann später noch einmal nach der Zerstörung Jerusalems und des Tempels im Jüdischen Krieg nach neuen Interpretationen des Kultes und des damit verbundenen Glaubens unter radikal veränderten Bedingungen suchte. Einen gewissen Höhepunkt erlebte die Spiritualisierung der Kultterminologie bei Philo von Alexandrien, bei dem die Kultrealien eine Moralisierung, Allegorisierung und Universalisierung erfuhren und auf ethische, seelische und geistige Prozesse im Mikrokosmos der menschlichen Seele oder im Makrokosmos des Universums übertragen wurden. Sie mutierten dadurch $\mathrm{zu}$ einem nur noch uneigentlichen und symbolischen Gleichnis für eine geistige Wirklichkeit, deren Grunddaten Philo in der platonischen und stoischen Philosophie fand.

Ein ähnliches Sprachphänomen lässt sich auch im Neuen Testament beobachten. Hier wurde aus dem Jerusalemer Tempel der Tempel der christlichen Gemeinde (1Kor 3,16f; 6,19; 2Kor 6,16; vgl. Mk 14,58) und aus den Opfern wurden pneumatische Opfer, wie

\footnotetext{
1 Von grundlegender Bedeutung war hier die Arbeit von H. Wenschkewitz, Die Spiritualisierung der Kultusbegriffe Tempel, Priester und Opfer im Neuen Testament (Angelos, Beiheft 4), Leipzig 1932. Auch die Dissertation von H.-J. Hermission aus dem Jahr 1961 trug den Titel »Die Spiritualisierung der Kultusbegriffe im Alten Testament «. In der veröffentlichten Version wanderte der ursprüngliche Titel dann in den Untertitel und der Begriff der Spiritualisierung wurde in Anführungszeichen gesetzt: Sprache und Ritus im altisraelitischen Kult. Zur "Spiritualisierung« der Kultbegriffe im Alten Testament, Neukirchen-Vluyn 1965.
} 
z.B. das "Lob« bzw. die "Frucht der Lippen", gute Werke und überhaupt das gehorsame, Gott hingegebene Leben des Christen (1Petr 2,5; Hebr 13,15f; Röm 12,1; vgl. Hebr 5,7). Schließlich wurde auch aus dem levitischen Priestertum Israels das allgemeine Priestertum aller Glaubenden, die sich durch den Glauben an und die Heiligung durch Jesus Christus in priesterlicher Gottunmittelbarkeit, -zugehörigkeit, -ähnlichkeit, Heiligkeit und Integrität erkannten (1Petr 2,5.9; Offb 1,5f; 5,9f; 20,6). ${ }^{2}$ Nach Hans Wenschkewitz ${ }^{3}$ seien so im Zuge der neutestamentlichen "Spiritualisierung" aus Realien vergeistigte Größen geworden.

Dieser Prozess wurde nun v.a. seit der Aufklärung auch für das Gebet angenommen, insofern es eine Wandlung von einem jüdischveräußerlichten und an den Kult gebundenen Ritual zu einem christlich-innerlichen, von allen »kultischen Fesseln« befreiten Geschehen erfahren habe. Entsprechend leitet Klaus Berger seinen TRE-Artikel zum Gebet im Neuen Testament mit den Worten ein: "Spätestens seit der Aufklärung leidet die Rezeption der neutestamentlichen Aussagen über das Gebet darunter, daß der allgemein religiöse, im weiteren Sinn kultische und von Magie nicht streng trennbare, vielmehr mit dem Offenbarungsgeschehen selbst verbundene Charakter des Gebets verkannt wird. Statt dessen hat man die Gebetsparänese puristisch vereinseitigt (besonders die gegen das >Plappern 'gerichtete), und ein durchaus 'werkgerechter Perfektionismus` zeigt an, daß auch hier >Religion` durch `Ethik` ersetzt wurde. $\ll^{4}$

Locus classicus für diese These ist natürlich Mt 6,5-8, wo Jesus im Rahmen der Bergpredigt seine Jünger lehrt, nicht öffentlich auf den Straßen zu beten wie die Heuchler, sondern im verschlossenen "Kämmerlein", und auch nicht plappernd viele Worte zu machen wie die Heiden, sondern sich auf das Wesentliche zu besinnen, das Jesus ihnen im anschließenden Vaterunser vorgibt. So zieht Emmanuel von Severus in einem 1953 erschienen Artikel in Erwägung, dass die Apostel in dem lauten Murmeln von Tora und Weisheitssprüchen »die Frömmigkeitshaltung der Pharisäer sahen, von der sie sich distanzieren wollten. Die jungen Christen sollten nicht nach deren veräußerlichter Art das Gesetz Gottes dauernd auf den Lippen haben und dabei die großen Gebote der Gottes- und Nächstenliebe

\footnotetext{
2 Siehe hierzu V. Gäckle, Allgemeines Priestertum. Zur Metaphorisierung des Priestertitels im Frühjudentum und Neuen Testament (WUNT 331), Tübingen 2014.

3 Wenschkewitz, Die Spiritualisierung der Kultusbegriffe Tempel, Priester und Opfer im Neuen Testament.

4 K. Berger, Art. Gebet IV. Neues Testament, in:TRE 12 (1984), 47-60, hier 48.
} 
vergessen. $\|^{5}$ Im Wandel von einem lauten Murmeln zu einer stillen Meditation sieht er einen Vorgang der »Entsinnlichung«, der prägend werden sollte für den christlichen Begriff der Meditation. ${ }^{6}$

Es stellt sich allerdings die Frage, ob zum einen die Begriffe der Spiritualisierung, Vergeistigung oder Entsinnlichung überhaupt geeignet sind, das genannte sprachliche Phänomen angemessen $\mathrm{zu}$ charakterisieren, und ob zum anderen dieses Phänomen für das Gebet überhaupt zutrifft. Wir werden uns deshalb zunächst einmal mit dem sprachlichen Vorgang der Übertragung von kultischen Begriffen auf andere und neue Sinnbereiche beschäftigen und anschließend fragen, inwiefern das Gebet daran teilhat.

\section{Spiritualisierung?}

In der zweiten Hälfte des 20. Jahrhunderts machte sich eine zunehmende Kritik am Begriff der "Spiritualisierung" bemerkbar. Mehr und mehr wurde deutlich, dass er in der Denktradition des deutschen Idealismus beheimatet und eng mit den entsprechenden philosophischen und theologischen Wertungen verbunden ist. So wurden geistige und damit immaterielle Ausdrucksformen der Frömmigkeit grundsätzlich höher bewertet als kultisch-materielle.Verbunden mit dem Evolutionsgedanken wurde die spiritualisierte Frömmigkeit als eine Weiter- und Höherentwicklung von kultischen und ritualisierten Formen bewertet. Entsprechend war die frühchristliche "Spiritualisierung» der Kultbegriffe für Wenschkewitz nur auf dem Boden der hellenistischen Gemeinden denkbar, nicht jedoch in Israel-Palästina, wo die Ausstrahlungen des Jerusalemer Kultes zu massiv waren. ${ }^{7}$ Diese Annahme erwies sich jedoch spätestens mit der Entdeckung der Qumran-Texte als ein Irrtum, denn bereits in diesen Texten findet sich dasselbe sprachliche Phänomen, das dort ganz unzweideutig nicht aus hellenistischem Hintergrund stammen kann. Für Unbehagen sorgte auch, dass die im Idealismus wurzelnde Prägung des Begriffs die spiritualisierten Begriffsempfänger der christlichen Gemeinde (Tempel, Priesterschaft) und ihrer Werke (Opfer) nur noch als "immateriell", "innerlich", "geistig", "himmlisch", "symbolisch" oder gar als "unwirklich" und "uneigentlich»

\footnotetext{
5 E. v. Severus, Das Wort meditari im Sprachgebrauch der Heiligen Schrift, in: Geist und Leben 26 (1953), 365-375, hier 375.

6 Ebd.

7 Wenschkewitz, Die Spiritualisierung der Kultusbegriffe Tempel, Priester und Opfer im Neuen Testament, 165.
} 
erscheinen ließ. Hinzu kam, dass der Begriff auch in konfessioneller Hinsicht eine polemische Konnotation enthielt, steckte doch in dem idealistischen Geist zu einem nicht geringen Maße auch der protestantische Geist der Tübinger Stiftler Hegel und Schelling. Mit der pejorativen Einschätzung kultischer Realien im Vergleich mit dem scheinbar vergeistigten Glauben der neutestamentlichen Gemeinde ging nicht selten auch eine anti-römische Attitüde einher, die den an das levitische Priestertum angelehnten, "veräußerlichten" römisch-katholischen Ritus ablehnte und einer spiritualisierten, protestantischen Innerlichkeit frönte. Während sich der Protestantismus mit dem scheinbar so kultkritischen Ethos der Propheten identifizierte, diente die veräußerlichte Frömmigkeit des levitischen Priestertums, das nomistisch an dem am Buchstaben normierten Kultus und Ritus festhielt, als Karikatur des römischen Katholizismus. Die so kreierten Stereotypen und Dichotomien hatten mit den biblischen Sachverhalten nur wenig zu tun, dafür umso mehr mit den reformationshistorischen Feindbildern.

Mit den begründeten Vorbehalten gegenüber dem Konzept der "Spiritualisierung intensivierte sich die Suche nach alternativen Bezeichnungen für das wahrgenommene Sprachphänomen. Es wurde eine Fülle von Begriffen vorgeschlagen wie "Entmaterialisierung«, "Entdinglichung", "Substitution", "Entsakralisierung", "Eschatologisierung", "Pneumatisierung", "Somatisierung", "Ethisierung", »Verbalisierung" oder »Kerygmatisierung «. ${ }^{8}$ An der Vielzahl der Vorschläge spiegelt sich die Aporie wider, dieses Sprachphänomen begrifflich adäquat zu fassen. In jüngerer Zeit hat sich der Begriff der "Metaphorisierung" durchgesetzt, weil er zum einen keine sekundären Wertungen in diesen antiken Sprachprozess einträgt und zum anderen vor dem Hintergrund der neueren Metaphertheorien sehr geeignet erscheint, das Wesentliche dieses Vorgangs zum Ausdruck zu bringen. Das sprachkreative Potential der Kultmetaphern versetzte die junge christliche Gemeinde in die Lage, eine vorher nicht aussagbare Erfahrung, wie z.B. die Erfahrung der Gegenwart Gottes im Kontext der urchristlichen Gemeindegottesdienste oder die im Glauben an Christus geschenkte Gottesunmittelbarkeit und -zugehörigkeit in eine neue Sprachform zu kleiden und dabei an die wesentliche Kontinuität zum Handeln Gottes im Alten Bund anzuknüpfen. Denn »Metaphern erinnern, um Neues zu sagen und sie erneuern, um Altes zu bewahren «". Durch die Metaphernbildung

\footnotetext{
8 Vgl. Gäckle, Allgemeines Priestertum, 9-13.

9 M. Buntfuss, Tradition und Innovation. Die Funktion der Metapher in der theologischen Theoriesprache (TBT 84), Berlin/New York 1997, 227.
} 
waren die ersten Christen in der Lage, die ihnen widerfahrende Offenbarung, die ihres Lebens im Geist und in der Gemeinde sprachlich adäquat $\mathrm{zu}$ beschreiben, was ohne Metaphern nicht möglich gewesen wäre.

Während die neutestamentliche Gemeinde auf diese Weise mit Hilfe der Kultmetaphern »Tempel«, "Opfer" und »Priester» in der Lage war, ihre Erfahrungen und ihr Selbstverständnis zu formulieren, gehört das Gebet nicht zu dieser sprachlichen Kategorie. Somit lassen sich die Wandlungen des Gebets im Neuen Testament weder mit den Begriffen der "Spiritualisierung" noch der "Metaphorisierung" angemessen beschreiben. Die dennoch wahrnehmbare Veränderung muss deshalb anders bestimmt werden.

\section{Kontinuitäten}

Die grundlegende Wandlung des Gebets im Neuen Testament ${ }^{10}$ besteht nicht in einer "Spiritualisierung " oder gar "Entsinnlichung«. Zwar haben wir nur begrenzte Informationen über die Gebetspraxis und -haltung der frühen Christen, doch vieles spricht dafür, dass die reiche Tradition der alttestamentlich-jüdischen Gebetsformen, -praktiken und -tageszeiten auch in den ersten judenchristlichen Gemeinden gepflegt wurden. ${ }^{11}$ So finden wir bei Jesus die alttestamentlichen Formen bzw. Gattungen des Lobpreises (Mt 11,25f), der Klage (Mt 27,46par), der Bitte (Mk 14,35-39par) und der Fürbitte (Lk 22,32; 23,34) wieder. ${ }^{12}$ Auch die jüdischen Gebetszeiten am

10 Der Autor ist sich durchaus bewusst, dass die Rede von »dem Gebet im Neuen Testament " den zahlreichen Nuancen, Akzentuierungen, Betonungen und Intentionen der verschiedenen neutestamentlichen Autoren nicht gerecht wird. Insbesondere K.H. Ostmeyer hat in seiner Habilitationsschrift, Kommunikation mit Christus und Gott. Sprache und Theologie des Gebetes im Neuen Testament (WUNT 197), Tübingen 2006, auf die Differenziertheit der neutestamentlichen Gebetsverständnisse aufmerksam gemacht. Vor dem Hintergrund der hier formulierten Fragestellung erscheint es dennoch gerechtfertigt, diese vergröberte Redeweise zu verwenden, um im begrenzten Rahmen dieses Beitrags auf die grundlegenden Elemente der Kontinuität und Wandlung hinzuweisen.

${ }_{11}$ Ostmeyer, Kommunikation mit Christus und Gott, 110: "Dass Paulus die üblichen [sc. jüdischen] Gebetszeiten einhielt ist wahrscheinlich. Jedenfalls lässt sich kein plausibler Grund nennen, warum er davon hätte abrücken sollen. Im Gegenteil wird er nach seiner Bekehrung die >Pflichtgebete in dem Bewusstsein gesprochen haben, dass sie erst jetzt von ihm im Blick auf den kýrios Iesoûs angemessen gebetet werden können.«

12 Ostmeyer, Kommunikation mit Christus und Gott, 110: "Der Unterschied zwischen jüdischem und paulinisch-christlichem Gebet liegt nicht in der Art des Betens. 
Morgen, Mittag und Abend lassen sich belegen. ${ }^{13}$ Vor allem an den Schlüsselstellen des Lukasevangeliums hat das Gebet die Funktion, die Kontinuität zwischen der Gotteserfahrung und dem Gottesverhältnis vor und nach Christus zu betonen. In der Apostelgeschichte dienen die Gebete dann dazu, die Kontinuität der Heilserfahrung und Gottesnähe nach Ostern und Himmelfahrt Jesu zum Ausdruck zu bringen. ${ }^{14}$

Es gibt auch keinen Hinweis darauf, dass sich aus theologischen Gründen wesentliche Veränderungen im Gebetsgestus vollzogen hätten. Die im Alten Testament belegten Gebetsgesten des Stehens (Gen 18,22; 1Sam 1,26; 1Kön 8,22), Niederkniens (1Kön 8,54), Sich-Niederwerfens (Gen 19,1; 24,26) und der Proskynese (Dtn 4,19; Ps 5,8; Dan 9,18; vgl. 2Sam 14,4.33; Jes 2,8; Dan 3,5-7), bei der Gesicht und Handflächen die Erde berühren, und das Ausstrecken bzw. Erheben der Hände (1Kön 8,22; Ps 141,2; Jes 1,15) sind auch im Neuen Testament belegt (vgl. aber Mt 2,11; 4,9; 8,2; 18,26; Apg 10,25; 21,5; 1Tim 2,8; Offb 19,10; 22,8; vgl. auch Mk 15,19; Lk 5,8; Eph 3,14). Wir finden auch die alttestamentliche Verknüpfung von Beten und Fasten wieder (Mk 2,19f; 9,29; Mt 6,16-18; Lk 2,37; Apg 10,9f; 13,1-3; 14,23; 1Kor 7,5).

Auch in Inhalt und Vielfalt der Alltagsgebete, die zu allen Zeiten ihren festen Ort neben den im Kult verorteten Gebeten hatten, lassen sich keine großen Veränderungen feststellen. Diese Gebete waren weder an den Kult, den Kultort oder an bestimmte Zeiten gebunden, sondern konnten überall und jederzeit an Gott gerichtet werden (vgl. 1Sam 25,32; 2Sam 15,31; Jes 38,1-3.9-20). In der Regel ist ihr Inhalt von einer bestimmten Not oder einem Bedürfnis geprägt (Gen 15,2f; 21,16f; 30,2; Ex 3,7; Num 12,13; Ri 16,28; 1Sam 1,10f; 2Sam 15,31; Jes 49,21). In zahlreichen Gebeten werden Alltagswünsche zum Ausdruck gebracht wie die Bitte um Gelingen, Vergewisserung und Führung (Gen 24,12-14; Ri 6,13), künftiger Beistand, Schutz und Vergebung (1Sam 26,24; 2Kön 5,18) auch im Blick auf Dritte (Gen 43,14; Ruth 1,8f; 2,12; 4,11; 1Sam 24,20;26,23;2Sam 14,17;24,3.23;1Kön 1,36f). All das begegnet uns

Juden und Christen kannten sowohl das Gebet als Grundhaltung gegenüber Gott als auch die besondere Wendung an Gott in Lob, Bekenntnis und Fürbitte (kursiv bei O.). So auch Gebauer, Art. Das Gebet im Neuen Testament, in: GBL I (21990), 414 416, hier 414: BBeten und Gebet stehen im NT, besonders bei Jesus und Paulus, in der Tradition des antiken Judentums", ähnlich ders., Art. Gebet III. Neues Testament, in: RGG 3 (2000), 488-491, hier 488.

${ }^{13}$ Mk 1,35; Apg 10,9; Mk 15,34par; 6,46f; vgl. auch Apg 3,1; 10,3.9.30; 16,25; Did 8,3 .

${ }^{14}$ Berger, Gebet IV. Neues Testament, 53f. 
auch im Neuen Testament in Form von Tischgebeten (1Tim 4,4f), Fürbittegebeten (Apg 12,12; Röm 15,30f; 2Kor 1,11), Gebeten in Abschiedssituationen (Apg 20,36; 21,5), Aussendungsgebeten (Apg 13,3), Heilungsgebeten (Jak 5,14) und sogar Rachegebeten (Offb $6,10)$.

Die Mahnung Jesu in Mt 6,5-8 darf sicher nicht als Aufforderung $\mathrm{zu}$ einer "Verinnerlichung" oder gar "Entsinnlichung " des Gebets verstanden werden. Es geht vielmehr zum einen um die Kritik einer von Jesus als Heuchelei bewerteten Gebetspraxis, die v.a. auf die Zurschaustellung der Frömmigkeit angelegt war und bei der das intime Gottesverhältnis des Beters in den Hintergrund trat $(6,5 \mathrm{f}){ }^{15}$ Dafür spricht der Kontext der Bergpredigt, in dem es in Mt 6,1-8 im Blick auf das Almosengeben und in 6,16-18 im Blick auf das Fasten um die Kritik veräußerlichter Frömmigkeitsformen geht, bei denen mehr die Außenwirkung auf die Betrachter als die persönliche Gottesbeziehung im Mittelpunkt steht. Die in der Antike und im Orient bis heute übliche Praxis des lauten, flehenden, murmelnden oder rufenden Betens wird damit ebenso wenig in Frage gestellt, wie das synagogale Gemeinschaftsgebet des Judentums. ${ }^{16}$ Zum anderen kritisiert Jesus eine heidnische Gebetspraxis, die das Ziel verfolgt, mit Hilfe langer und intensiver Gebete die jeweilige(n) Gottheit(en) in Richtung auf das erbetene Anliegen hin zu manipulieren, gemäß dem Grundsatz »viel hilft viel«. Möglicherweise wird hier auch eine ekstatische Form des Gebets kritisiert, die in der paganen Mystik bekannt war und bei der durch ständige Wiederholung desselben Gebets ein Zustand der Ekstase hergestellt wurde. ${ }^{17}$ Eine solche Gebetshaltung entspräche nicht dem Gott, den Jesus als seinen Vater vorstellt. Er ist nicht durch ein bestimmtes Gebetsverfahren oder bestimmte Techniken manipulierbar.Vielmehr weiß er schon vorher um das Anliegen des Beters, und mit dem folgenden Vaterunser (V. 9-13) bestimmt Jesus das Gebet als eine Kommunikation zwischen einem Vater und seinen Kindern.

\footnotetext{
${ }_{15}$ Vgl. hierzu U. Luz, Das Evangelium nach Matthäus. Mt 1-7 (EKK I/1), Düsseldorf, Zürich, Neukirchen-Vluyn ${ }^{5} 2002$, 424f.

${ }^{16}$ Ebd., 426, sowie 432: »Synagoger ist zum Typos für das von der christlichen Kirche überwundene Negative geworden, ein Typos allerdings, mit dem sich die wirklichen Juden jederzeit zu identifizieren hatten. Von hier aus ist es dann nicht unbegreiflich, wenn immer wieder einmal - von Chromatius bis zu Adolf Schlatter - die Pharisäer bzw. >die Judenschaft zu denjenigen werden, deren wortreiches Gebet Jesus geißle.»

${ }^{17}$ Berger, Gebet IV. Neues Testament, 50.58; vgl. Sir 7,14 und Jos Bell 6,306.
} 


\section{Wandlungen}

Die wesentlichen Veränderungen des Gebets kommen im Neuen Testament nicht in der Gebetsform, dem -gestus oder den -inhalten zum Ausdruck, sondern in der Gebetsanrede, im Gottesverhältnis, im Bezugsrahmen und im Inhalt der Dankgebete: "Durch sein Heilshandeln in Jesus Christus erschließt sich Gott als der, zu dem Jesus betet und beten lehrt und zu dem die Gläubigen aufgrund ihres Angenommenseins mit einer vorher nie dagewesenen Gewißheit beten können. ${ }^{18}$

\subsection{Gebet »durch Christus» und »im Geist»}

In Christus erkennen die ersten Christen nicht nur, dass Gott durch ihn bzw. in seinem Kommen, Sterben und Auferstehen zu ihrem Heil gehandelt hat, sondern dass er als der Auferstandene und Erhöhte auch der eigentliche Ermöglichungsgrund, Mittler und vereinzelt sogar Adressat ${ }^{19}$ ihrer Gebete ist. Entsprechend betet Paulus »durch Christus" und "in Christus« zu Gott (Röm 1,8; 5,11; 7,25; 2Kor 1,20; Kol 3,17). Im Vordergrund steht bei ihm folglich das Dankgebet für das erfahrene Heilshandeln in Christus. "Das Heilsgeschehen am Kreuz bildet die von Gott gesetzte Grundlage alles

\footnotetext{
18 Gebauer, Das Gebet im Neuen Testament, 414; vgl. auch Ostmeyer, Kommunikation mit Christus und Gott, 115: „Für Paulus ist nicht eine besondere Gebetsform das Spezifikum christlicher Existenz, sondern die durch Christi Tod und Auferweckung ermöglichte Beziehung des Gläubigen zu Gott, die sich in den verschiedensten Arten zu beten äußern kann.»

${ }_{19}$ Apg 7,59f; 1 Kor 16,22; Offb 22,20; 2Kor 12,8; vgl. auch 1Tim 1,12. Grundsätzlich richten sich auch im Neuen Testament die Gebete der Glaubenden an »Gott« bzw. "den Vater«, vgl. hierzu J.D.G. Dunn, Did the first Christians worship Jesus?, London/ Louisville (KY) 2010, 37: "The most explicit prayer language is used exclusively of prayer to God. Jesus himself is remembered as regularly praying to God and giving instruction on prayer to God. [...] If, speaking with tightly focused precision, sprayer as such was not usually made to Jesus in the worship of the first Christian congregations, at least he was regarded as one, sitting at God's right hand, who could be and was called upon, and to whom appeal could be made." Allerdings ist es nunmehr eben derVater Jesu Christi und der Gott, der sich in Christus letztgültig offenbart hat, der hier in der Gebetsanrede angesprochen wird. Umgekehrt werden Christen als Menschen bezeichnet, "die den Namen des Herrn Jesus Christus anrufen" (Apg 2,21; 9,14.21; 22,16; Röm 10,12-14; 1Kor 1,2; 2Tim 2,22). Dies wurde offensichtlich als ein Charakteristikum der frühen Jesus-Bewegung wahrgenommen.
} 
paulinischen Betens. Unter Absehung vom Kreuz Christi ist Beten, wie es sich in den paulinischen Briefen darstellt, nicht möglich. $\otimes^{20}$

Demgegenüber tritt die sowohl in den Psalmen wie bei Jesus häufig thematisierte Frage der Gebetserhörung auffallend in den Hintergrund. Wir finden bei Paulus die geradezu unerhörte Gewissheit, dass Gott den betenden Menschen erhört, wenn auch auf eine Art und Weise, die dem Beter selbst möglicherweise verborgen bleibt (Röm 8,28). So kann er vordergründiger Nichterhörung seiner Gebete einen tieferen Sinn abgewinnen (2Kor 12,7-10) oder sich und andere zu noch intensiverer und anhaltenderer Fürbitte motivieren (Röm 15,30; 2Kor 1,11). Vor dem Hintergrund der Offenbarung der Liebe Gottes in Kreuz und Auferstehung Christi (Röm 5,8) ist sich Paulus gewiss, dass er jenseits aller Lebensfügungen untrennbar mit Gott verbunden ist und bleibt (Röm 8,38f). "Deshalb ist es ein Charakteristikum paulinischen Betens, dass für den Apostel die Erfüllung einzelner Gebetsanliegen nicht entscheidend ist. [...] Die Gebetsbeziehung kann durch die Nichterfüllung einzelner Gebete nicht gestört werden. ${ }^{21}$ Der Apostel erkennt in Christi Heilswerk die grundlegende Erhörung aller menschlichen Hoffnungen und Sehnsüchte (vgl. 2Kor 1,20), die zwar noch nicht sichtbar geworden, aber in Christus schon jetzt eine geistliche Wirklichkeit ist (vgl. 2Kor 4,16.18; 5,7 u.ö.).

Neu ist auch die Verbindung des Gebets mit dem Heiligen Geist, die im Judentum so unbekannt war. Es ist der Geist, der die Glaubenden zum Gebet treibt (Röm 8,14f) und in dessen Macht das Gebet sich vollzieht (Eph 5,18-20; 6,18f). Der Geist vertritt die Glaubenden aufgrund ihrer irdisch-menschlichen Schwachheit vor Gott (Röm 8,26f) und lässt sie das neue Gottesverhältnis der Kindschaft erfahren (Röm 8,15; Gal 4,6f), das auch in der von Jesus völlig neu eröffneten Abba-Anrede Gottes seinen Ausdruck findet (vgl. Mk 14,36). So ist das Gebet ein »unmittelbarer Ausdruck der durch Jesus eröffneten neuen Gottesbeziehung des Menschen. $\star^{22}$

\section{2 »In Christus« statt »im Tempel«}

Eine wesentliche Wandlung betrifft nun aber die Verortung des Gebets im Kult bzw. Tempel. Seit der Frühzeit der alttestamentlichen Überlieferung war das Gebet nicht nur in der Alltagsfrömmigkeit,

\footnotetext{
${ }^{20}$ Vgl. R. Gebauer, Das Gebet bei Paulus. Forschungsgeschichtliche und exegetische Studien, Gießen 1989, 217.

${ }^{21}$ Ostmeyer, Kommunikation mit Christus und Gott, 108.

22 Gebauer, Gebet III. Neues Testament, 488.
} 
sondern v.a. auch im Kultus und am Kultort, später dann am Tempel und im Tempelgottesdienst verortet. Dort war zunächst auch für Jesus und die Jerusalemer Urgemeinde der genuine Ort des Betens, der Versammlung und Verkündigung. ${ }^{23}$ Umso überraschender ist es, dass mit der Vertreibung der jungen Gemeinde aus dem Tempel und schließlich aus Jerusalem keine theologische Krise der jungen Bewegung einherging, wie dies bei den exilierten Juden in Babylon 600 Jahre vorher der Fall gewesen war. Die junge Jesus-Bewegung zog vielmehr vom Tempel in die Privathäuser - und später in die Diaspora nach Antiochien, Alexandrien, Kleinasien und anderen Orten - und betete dort, ohne dass dies tiefere Spuren einer theologischen Bewältigungsphase hinterlassen hätte.

Das Verhältnis zum Tempel war in der jungen Gemeinde von Unabhängigkeit geprägt. Diese Unabhängigkeit spiegelt sich am deutlichsten bei Paulus wieder: Auf der einen Seite spielt der Jerusalemer Tempel als zentrale Kultinstitution des antiken Judentums für das paulinische Denken keine Rolle mehr. ${ }^{24}$ Nirgendwo in den erhaltenen Paulusbriefen des Neuen Testaments finden wir eine Reflexion über das Verhältnis des Heilshandelns Gottes in Jesus Christus zum Jerusalemer Tempel. Der einzige wirklich relevante Beleg ist der sühnetheologische locus classicus Röm 3,25f, der gleichzeitig zeigt, dass Paulus die Kultbegriffe in großer Freiheit metaphorisch verwenden konnte.

Auf der anderen Seite darf dies nicht als eine Kritik des Jerusalemer Tempels missverstanden werden. Anders als z.B. in den Qumrantexten konstruiert Paulus nirgendwo einen expliziten Gegensatz zwischen dem »Tempel der Gemeinde " (1Kor 3,16f; 2Kor 6,16) und dem Jerusalemer Heiligtum..$^{25}$

Der Tempel war für Paulus nach wie vor ein legitimer Ort jüdischer Anbetung und Gottesverehrung, aber - und das ist für den (Diaspora!-) Judenchristen und Apostel Paulus höchst relevant -

\footnotetext{
${ }^{23}$ Lk 24,53; Apg 2,46; 3,1-26; 4,1ff; 5,12.19-21.42; 22,17.

24 P.Wick, Die urchristlichen Gottesdienste. Entstehung und Entwicklung im Rahmen der frühjüdischen Tempel-, Synagogen- und Hausfrömmigkeit (BWANT 150), Stuttgart 22003, 192: »Der Jerusalemer Kult ist für ihn [sc. Paulus] eine Größe, die er nicht ersetzen will, die aber für ihn als Jesusanhänger nicht mehr im Zentrum seines religiösen Bemühens steht. [...] [Z]ur Umschreibung der gemeindlichen Versammlungen und Gottesdienste gebraucht Paulus bezeichnenderweise keine Kultmetaphorik. Versammlungen und Mahl- und Gebetsgemeinschaften sind weder Kultersatz noch werden sie in Richtung Kult aufgewertet noch durch Metaphorik in dessen Nähe gerückt. Die gottesdienstlichen Versammlungen der Ekklesia haben keinen kultischen Anspruch.»

${ }^{25}$ Gäckle, Allgemeines Priestertum, 370-373.
} 
nicht mehr der einzige. Indem Christus den eschatologischen "Zugang" (vgl. Röm 5,2) zu Gott für Juden und Heiden eröffnet hat, können diese nun "im Glauben" (Röm 3,25) und "in Christus" in die Gegenwart Gottes treten und finden in der Gemeinde der Glaubenden den eschatologischen Ort der Gottesbegegnung (1Kor 3,16f; 2Kor 6,16).

Deshalb war der Tempel nie ein entscheidender Teil der religiösen Identität der paulinischen Gemeinden; diese wurde ausschließlich über das Verhältnis zu Christus begründet (vgl. 2Kor 5,17; Gal 2,20). Auch wenn Jerusalem und die dortige Gemeinde für Paulus immer der heilsgeschichtliche Ausgangspunkt des Evangeliums und seiner apostolischen Sendung blieb (vgl. Röm 15,19.25; sowie 11,26) und er deshalb auch die von ihm gegründeten Gemeinden in einer bleibenden Verpflichtung gegenüber der Jerusalemer Gemeinde sah (Röm 15,26f; vgl. 1Kor 16,1-4; 2Kor 8-9), so fühlten sich die heidenchristlichen Gemeinden (und letztlich auch Paulus) niemals intensiver dem Tempel verpflichtet. ${ }^{26}$ An die Stelle des Tempels war Christus selbst und die Gemeinde als sein Leib (1Kor 12,12ff; Röm 12,4f) getreten. Vor diesem Hintergrund muss auch die lokale Bedeutung der Wendung "in Christus ${ }^{27}$ verstanden werden: »It was belonging to Christ, being sin Christ<, being the body of Christ, that provided the Christian equivalent to the holy place. If the Jerusalem Temple was the key identity marker for Jews both in the land of Israel and in the Jewish diaspora, then Christ himself was the defining identity marker for the first Christians (Christ-ians). Christ himself functioned in effect as the Christian sacred space. $\|^{28}$

Eine ähnliche Loslösung des Gebets vom Kult findet auch im Blick auf Zeiten, Opfer und Personen statt. ${ }^{29}$ So sehr der Sabbat zu den jüdischen identity markers gehörte (vgl. z.B. Jes 56,6), so wenig

\footnotetext{
${ }^{26}$ Wick, Die urchristlichen Gottesdienste, 192: »Paulus bemüht sich nicht, die jüdischen Gläubigen vom Jerusalemer Tempelkult abzubringen und diesen durch einen neuen, noch höheren Kult zu ersetzen. Das entspräche überhaupt nicht seinem argumentativen und theologischen Interesse. Allerdings bindet er die bekehrten Heiden auch nicht an den Tempel. In der Diaspora kamen diese bei einem Übertritt zum Glauben an Jesus in eine jüdische Gemeinschaft, die nur einen fernen Tempel hatte. Nachfolge Christi hieß für sie, im Gegensatz zu ihren hellenistischen Landsleuten radikal auf jeden Opferkult zu verzichten, da eine Teilnahme an hellenistischen Kulten aus Glaubens- und Gesetzesgründen nicht mehr möglich war und der Jerusalemer Tempel gleichfalls nur eine ferne Größe blieb.«

${ }^{27}$ Röm 6,11.23; 8,1f.39; 9,1; 12,5; 15,17; 16,3.7.9f; 1Kor 1,2.4.30; 3,1; 4,10.15.17.22.31; 16,24; 2Kor 2,14.17; 3,14; 5,17.19; 12,2.19; Gal 2,4; 3,14.28; 5,6; Phil 1,1.13.26; 2,1;3,14; 4,7.19; 1Thess 1,1;2,14;4,16;5,18; Phlm 8 u.v.a.m.

${ }^{28}$ Dunn, Did the first Christians worship Jesus?, 47.

${ }^{29}$ Vgl. ebd., 48-56.
} 
orientierten sich Paulus und die frühen Gemeinden an ihm (vgl. Röm 14,5f; evtl. auch Kol 2,16). An die Stelle des Sabbats als der Zeit des Gottesdienstes und gemeinsamen Gebets trat der Sonntag als Auferstehungstag des Herrn (Mk 16,2; Apg 20,7; 1Kor 16,2; Ign Magn 9,1) und dies galt wohl auch, wie Offb 1,10 zeigt, für judenchristliche Gemeinden. Mit dem Sonntag standen auch nicht mehr der Auszug aus Ägypten als Grunddatum Israel im Mittelpunkt der Erinnerung, sondern Tod und Auferstehung Jesu Christ als Grunddatum der Gemeinde.

Mit dem "Sein in Christus" erübrigte sich auch die Rolle der Priester als Mittler zwischen Gott und Menschen bzw. Menschen und Gott, die ihnen in nahezu allen mediterranen Kulturen und Religionen zukam. ${ }^{30}$ Aufgrund der im Glauben eröffneten Unmittelbarkeit und Zugehörigkeit zu Gott und der Heiligkeit und Integrität vor Gott (vgl. 1Kor 1,2; 1,30; 6,11) konnten einzelne neutestamentliche Autoren die Christen selbst als Priester bzw. Priesterschaft bezeichnen (vgl. 1Petr 2,5.9; Offb 1,6; 5,10;20,6), die keines mediatorischen Dienstes einer dafür spezialisierten Kaste mehr bedurften.

Schließlich löste sich auch der nicht nur alttestamentliche, sondern gemeinantike Konnex zwischen kultischem Gebet und Tieropfern, weil nach Röm 3,25 und Hebr 9,1-10,18 Christus nun selbst nicht nur als der eschatologisch offenbarte Kultort, sondern auch als das eine eschatologisch gültige und alle anderen Opfer übertreffende und damit ablösende Opfer "für unsere Sünden" (1Kor 15,3) verstanden wurde. ${ }^{31}$ Die Gebete selbst wurden nun in Anknüpfung an alttestamentliche und jüdische Formulierungen (z.B. Ps 50,14.23; $141,2)$ als metaphorische Opfer verstanden (1Petr 2,5; Hebr 13,15; Offb 8,3f).

Diese vollständige Lösung des gottesdienstlichen Gebets vom Tempelkult mit seinen von einer Priesterschaft dargebrachten Op-

\footnotetext{
${ }^{30}$ Vgl. hierzu Gäckle, Allgemeines Priestertum, 19-42.

${ }^{31}$ Zwar löst Paulus in Apg 21,26 durch ein Opfer die Gelübde von vier Männern aus. Eine soteriologische Absicht verbindet er damit aber nicht mehr. Diese Opfer sind Teil seiner jüdischen Identität, die ihm die Freiheit in Christus lässt. Wir finden jedoch im Neuen Testament keinen Hinweis mehr darauf, dass Christen Opfer mit soteriologischer Intention dargebracht hätten. Erst im Ebionäerevangelium in Frg. 6 = Epiphanius Pan 30,16,4-5; vgl. auch 28,1,4 und 29,1,6; sowie PsClem Hom 3,56,4 und Recogn 1,39, findet sich eine Polemik gegen die (judenchristliche?) Teilnahme an Opfern: "Ich bin gekommen, die Opfer abzuschaffen, und wenn ihr nicht ablasst zu opfern, wird der Zorn von euch nicht ablassen. "Ob dies als ein Reflex auf eine fortgesetzte Teilnahme (von einzelnen Mitgliedern?) der Jerusalemer judenchristlichen Gemeinde am Opferkult gewertet werden darf, muss aber offen bleiben.
} 
fern war einzigartig in der uns bekannten antiken Welt: »Unlike any other cult or club, there was no sacred space in which they met, nor far-off Temple towards which they directed their worship. Their sacred time was different and distinct, their sacred meal allowed no comparison or competitor. There were no priests present to officiate and to render their meals acceptable or their worship possible. No sacrifices were offered; there were no libations to any god. Onlookers might well wonder whether this was a cult, whether their gatherings were religious, as the practice of religion was generally understood. (12 $^{32}$

\section{Abgrenzungen}

Aus den beschriebenen Wandlungen des Gebets vor dem Hintergrund der Christusoffenbarung resultieren vereinzelt auch dezidierte Abgrenzungen. Dies ist vor allem im Johannesevangelium zu beobachten. ${ }^{33}$ Dort markiert der vierte Evangelist mit Hilfe seiner subtilen und nuancierten Verwendung von Begriffen eine deutliche Grenze zwischen jüdischem und christlichem Beten. Dies wird schon dadurch deutlich, dass die traditionellen jüdischen Gebetsbegriffe überhaupt nicht vorkommen: "The terms which are connected with the cult of the temple - that means the majority of >classical prayer terms - have been erased. \$ $^{34}$

In Joh 4,20-24 antwortet Jesus auf die Frage der Samaritanerin nach dem rechten Ort der Anbetung, dass sich die wahre Anbetung nicht an einem bestimmten geographischen Ort vollzieht, sondern "im Geist und in der Wahrheit". Dabei ist auffallend, dass Johannes bei der Frage der Frau, die sich auf die Alternative zwischen dem Jerusalemer Tempel und dem samaritischen Alternativtempel auf dem Garizim bezieht, ${ }^{35}$ weder den Begriff des »Tempels« noch des "Kultes" oder des "Gottesdienstes" erwähnt. Die Vermeidung des

\footnotetext{
${ }^{32}$ Dunn, Did the first Christians worship Jesus?, 57 (kursiv bei D.).

${ }^{33}$ Siehe hierzu K.-H. Ostmeyer, Prayer as Demarcation. The Function of Prayer in the Gospel of John, in: H. Klein/V. Mihoc/K.-W. Niebuhr (Hg.), Das Gebet im Neuen Testament.Vierte europäische orthodox-westliche Exegetenkonferenz in Sâmbata de Sus, 4.-8. August 2007 (WUNT 249), Tübingen 2009, 233-247.

${ }^{34}$ Ostmeyer, Prayer as Demarcation, 247.

${ }^{35}$ Vgl. dazu T. Wardle, The Jerusalem Temple and Early Christian Identity (WUNT II/291), Tübingen 2010, 99-120; I. Hjelm, Jerusalem's Rise to Sovereignity. Zion and Gerizim in Competition, London 2004, sowie den Sammelband von J. Frey/U. Schattner-Rieser/K. Schmid (Hg.), Die Samaritaner und die Bibel/The Samaritans and the Bible. Historische und literarische Wechselwirkungen zwischen biblischen und samaritanischen Traditionen/Historical und Literary Interactions between Bibli-
} 
Naheliegenden dürfte kein Zufall sein. Das »betonte Verschweigen« des Tempels muss als subtile Abgrenzung vom jüdischen Ort des Betens verstanden werden, der in den Augen des vierten Evangelisten durch Jesus ersetzt wurde. ${ }^{36}$ Doch mit dem Ort des Tempels findet auch eine Abgrenzung zu einem Gottesverhältnis statt, das Christus nicht anerkennt: »The renouncement of traditional, ritual terms in the Gospel of John represents the renunciation of the sold relationship with God, which is expressed by exactly these terms. ${ }^{37}$

\section{Fazit}

Der Glaube an Christus veränderte die Gebete der ersten Christen trotz aller formalen und gestischen Kontinuitäten weit umfassender, als dies auf den ersten Blick wahrgenommen werden kann. Christus wird nicht nur zum Mittler, Ermöglicher und vereinzelt sogar (Mit) Adressat ihres Lobs und ihrer Gebete, sondern auch zum Raum, „in" dem sie sich in der Gegenwart Gottes wussten. In diesem Raum eröffnete sich nicht nur die Gelegenheit zur Zwiesprache mit Gott, sondern eine "mit Gott kommunizierende Existenz « ${ }^{38}$. In diesem betenden Sein "vor", "bei« und "mit Gott« "in Christus" und "im Geist" kommt der Glaubende letztlich auch zur eschatologischen Erfüllung all seiner in der Gegenwart noch unbeantworteten Fragen und Gebetsanliegen. »[I]n der Überzeugung, dass nur in Christus eine Kommunikation mit Gott möglich sei, liegt einer der wesentlichen Unterschiede zwischen paulinisch-christlicher und jüdischer Gebetsauffassung. ${ }^{39}$ Das christliche Gebet war aus dieser Perspektive ohne Christus nicht mehr denkbar und ein Gebet an Gott, das nicht "durch und in Christus" und nicht "im Geist " gebetet wird, würde die heilsgeschichtliche Offenbarung dieses Gottes ignorieren und vom Heil ausschließen.

- Prof. Dr. Volker Gäckle ist Professor für Neues Testament an und Rektor der Internationalen Hochschule Liebenzell und Privatdozent an der Theologischen Fakultät der Universität Zürich.

cal and Samaritan Traditions (Studia Samaritana 7), Berlin 2012, hier v.a. der Beitrag von M. Kartveit, The Second Temple and the Temple of the Samaritans, 67-80.

${ }^{36}$ Ostmeyer, Prayer as Demarcation, 238, 241. In ähnlicher Weise interpretiert er, 239f, auch die Nichterwähnung des Tempels in Joh 12,20-23, wo die Griechen zur "Anbetung" nach Jerusalem kommen.

${ }^{37}$ Ebd., 247.

${ }^{38}$ Ostmeyer, Kommunikation mit Christus und Gott, 365.

${ }^{39}$ Ebd., 117. 